\title{
The use and evaluation of self-regulation techniques can predict health goal attainment in adults: an explorative study
}

Jolien Plaete, Ilse De Bourdeaudhuij, Maite Verloigne, Geert Crombez

Background. Self-regulation tools are not always used optimally, and implementation intention plans often lack quality. Therefore, this study explored participants' use and evaluation of self-regulation techniques and their impact on goal attainment.

Methods. Data were obtained from 452 adults in a proof of concept (POC) intervention of 'MyPlan', an eHealth intervention using self-regulation techniques to promote three healthy behaviours (physical activity(PA), fruit intake, or vegetable intake). Participants applied self-regulation techniques to a self-selected health behaviour, and evaluated the self-regulation techniques. The quality of implementation intentions was rated by the authors as a function of instrumentality (instrumental and non-instrumental) and specificity (non-specific and medium to highly specific). Logistic regression analyses were conducted to predict goal attainment.

Results. Goal attainment was significantly predicted by the motivational value of the personal advice (OR:1.86), by the specificity of the implementation intentions (OR:3.5), by the motivational value of the action plan (OR:1.86), and by making a new action plan at follow-up (OR:4.10). Interaction-effects with behaviour showed that the specificity score of the implementation intention plans (OR:4.59), the motivational value of the personal advice (OR:2.38), selecting hindering factors and solutions(OR:2.00) and making a new action plan at follow-up (OR:7.54) were predictive of goal attainment only for fruit or vegetable intake. Also, when participants in the fruit and vegetable group made more than three plans, they were more likely to attain their goal (OR:1.73), whereas the reverse was the case in the PA group (OR:0.34).

Discussion. The chance that adults reach fruit and vegetable goals can be increased by including motivating personal advice, self-formulated action plans, and instructions/strategies to make specific implementation intentions into eHealth interventions. To increase the chance that adults reach short-term PA goals, it is suggested to keep eHealth PA interventions simple and focus only on developing a few implementation intentions. However, more research is needed to identify behaviour change techniques that can increase health goal attainment at long-term. 
1 The use and evaluation of self-regulation techniques can predict health goal

2 attainment in adults: an explorative study

3 Jolien Plaete ${ }^{1}$, Ilse De Bourdeaudhuij ${ }^{1}$, Maïté Verloigne ${ }^{1}$, Geert Crombez ${ }^{2}$

4

${ }^{1}$ Department of Movement and Sports Sciences, Ghent University, Belgium

${ }^{2}$ Department of Experimental-Clinical and Health Psychology, Ghent University, Belgium

8 Corresponding author:

9 Jolien Plaete ${ }^{1}$

Watersportlaan 2, 9000 Gent, Belgium. Tel.: +32 92646363

Email address: jolien.plaete@ugent.be

12

\begin{abstract}
Background. Self-regulation tools are not always used optimally, and implementation intention plans often lack quality. Therefore, this study explored participants' use and evaluation of selfregulation techniques and their impact on goal attainment.

Methods. Data were obtained from 452 adults in a proof of concept (POC) intervention of 'MyPlan', an eHealth intervention using self-regulation techniques to promote three healthy behaviours (physical activity(PA), fruit intake, or vegetable intake). Participants applied selfregulation techniques to a self-selected health behaviour, and evaluated the self-regulation techniques. The quality of implementation intentions was rated by the authors as a function of instrumentality (instrumental and non-instrumental) and specificity (non-specific and medium to highly specific). Logistic regression analyses were conducted to predict goal attainment.

Results. Goal attainment was significantly predicted by the motivational value of the personal advice (OR:1.86), by the specificity of the implementation intentions (OR:3.5), by the motivational value of the action plan (OR:1.86), and by making a new action plan at follow-up (OR:4.10). Interaction-effects with behaviour showed that the specificity score of the implementation intention plans (OR:4.59), the motivational value of the personal advice (OR:2.38), selecting hindering factors and solutions(OR:2.00) and making a new action plan at follow-up (OR:7.54) were predictive of goal attainment only for fruit or vegetable intake. Also, when participants in the fruit and vegetable group made more than three plans, they were more likely to attain their goal (OR:1.73), whereas the reverse was the case in the PA group (OR:0.34). Discussion. The chance that adults reach fruit and vegetable goals can be increased by including motivating personal advice, self-formulated action plans, and instructions/strategies to make specific implementation intentions into eHealth interventions. To increase the chance that adults reach short-term PA goals, it is suggested to keep eHealth PA interventions simple and focus
\end{abstract}


37 only on developing a few implementation intentions. However, more research is needed to 38 identify behaviour change techniques that can increase health goal attainment at long-term.

39

40

41

\section{Introduction}

Physical activity (PA) and a varied diet with fruits and vegetables are associated with decreased risk of cardiovascular diseases and cancer(Lock et al. 2005; WHO 2009; WHO 2010). Therefore, adults are recommended to perform at least 30 minutes of PA at moderate to vigorous intensity on most, preferably all days of the week, and to consume at least $400 \mathrm{~g}$ of fruit and vegetable per day(Haskell et al. 2007). However, many adults do not meet these recommendations(WHO 2003). Despite the efforts to promote these health behaviours in adults, fruit and vegetable intake have been decreasing, and PA levels have remained the same since 2008 in Belgium. A recent meta-analysis focusing on these health behaviours indeed stated that changing unhealthy lifestyle is difficult, and there is room for improvement(Hallal et al. 2012). In previous computer-tailored interventions grounded in social-cognitive theories (e.g. Theory of Planned Behaviour), tailored feedback was given on motivational determinants such as awareness, knowledge, subjective norm and outcome expectations. Based on the individuals' scores on scales that measure these determinants, participants were provided with feedback that included a number of tips and suggestions for increasing or maintaining health behaviour(De Vries \& Brug 1999; Kroeze 2006 ; Vandelanotte 2003). For example, participants who had a positive attitude regarding PA, but who were not aware that they were not sufficiently physically active, mainly received information about PA norms and on how to increase PA levels. Whereas, participants who had negative attitudes, got tailored feedback on advantages of PA. However, interventions grounded in social-cognitive theories often only target determinants that are important during the early stages of behaviour change. They are also often more effective in 
62 changing intentions than in changing behaviour(Hagger et al. 2012; Sheeran et al. 2005),

63 resulting in a so-called intention-behaviour "gap". This gap can be targeted by also adopting self-

64 regulation techniques. One useful framework in this context is the Health Action Process

65 Approach model that includes both pre-intentional processes that lead to a behavioural intention

66 and post-intentional processes that lead to the actual health behaviour(Schwarzer 2008). The

67 model states that individuals first have to become conscious of their own health behaviour and

68 have to be become motivated to change their behaviour, whereafter they have to initiate the new

69 health behaviour to bridge the gap between intentions and behaviour. This can be achieved by

70 defining specific action plans about 'when', 'where', and 'how' to perform the health behaviour,

71 and by stating implementation intentions in which strategies to initiate the action are stated (i.e.

72 "If situation $\mathrm{Y}$ is encountered, then I will initiate goal-directed behaviour $\mathrm{X}$ ")(Gollwitzer \&

73 Sheeran 2006). People may also make coping plans in which they state how to cope with

74 anticipated barriers and problems that may hinder goal attainment(Bélanger-Gravel et al. 2013;

75 Schwarzer 2008; Sniehotta et al. 2006). Research has shown that interventions that applied self-

76 regulation techniques (i.e. specific goal setting, implementation intentions, providing feedback

77 on performance, prompting review of behaviour goals, social support and self-monitoring) were

78 more effective in changing health behaviour than other interventions that only targeted pre-

79 intentional determinants in tailored feedback2014 2009(Broekhuizen et al. 2012; Lara et al.

80 2014; Michie et al. 2009; Morrison et al. 2012).

81 Therefore, based on previous intervention studies(Spittaels et al. 2007; Springvloet et al. 2014;

82 van Genugten et al. 2010; Vandelanotte 2003) and the meta-analyses of Michie et al. (2009) and

83 Gollwitzer and Sheeran (2006) we integrated different behaviour change techniques into a novel

84 self-regulation eHealth intervention that targets both pre-intentional and post-intentional 
85 processes. Pre-intentional processes were targeted with tailored feedback. Post-intentional 86 processes were addressed with action planning, implementation intentions, problem solving, 87 sharing action plans with friends/family for social support, stimulating self-monitoring and goal 88 evaluation and adjustment. 'MyPlan' provided the opportunity to select one out of three health behaviours (fruit, vegetables and PA), provided tailored feedback to prompt intention formation, helped adults to set personal goals, and guided them to plan their behaviour and anticipate barriers and hindering situations during goal pursuit. Other studies, that integrated planning tools, have shown that many tools (e.g. action planning, implementation intentions) are used suboptimally by participants(Springvloet et al. 2014; van Genugten 2011; van Osch et al. 2010). For example, Michie et al. (2004) found that more than one-third of pregnant women intending to undergo prenatal screening did not formulate implementation intentions for attending or making an appointment despite being prompted to do so(Michie et al. 2004). Furthermore, when selfregulation tools were used, participants did not optimally apply them. Van Osch et al. (2010) reported that plans to promote smoking cessation that are relatively broad and non-specific resulted in less successful behavioural change. Ziegelmann, Lippke and Schwarzer (2006) evaluated completeness of fruit and vegetable plans developed by young, middle-aged and older patients in a rehabilitation clinic(Ziegelmann et al. 2006). They found that plans that were incomplete (lacking action planning or coping planning) were associated with less physical activity during rehabilitation at 6 months post-test. This shows that self-regulation techniques are perhaps not always feasible, or are difficult to apply. Therefore, it is important to test whether behaviour change techniques that are included in new interventions are acceptable and feasible 107 for the intended target population and to examine the quality of action plans2001(Tones \& 
108 Tilford 2001). The first aim of this study was to evaluate whether the use of several self-

109 regulation techniques (e.g. selecting hindering factors and solutions, monitor behaviour) and

110 feasibility of the self-regulation techniques (e.g. the difficulty experienced when making an

111 action plan, perceived feasibility of the action plan) could predict goal attainment in the target

112 group (i.e. adults over 18 years). Second, we rated the quality of implementation intention plans

113 and evaluated if the total number of instrumental plans and the specificity score of the

114 implementation intention plans could predict goal attainment. Finally, the moderating effect of

115 the selected behaviour (fruit intake, vegetable intake, PA) on the predictions of use and

116 feasibility rating of the self-regulation techniques on goal attainment was examined, as previous

117 research showed that the effect of behaviour change techniques, may vary for different

118 behaviours(Bélanger-Gravel et al. 2013).

119 Methods

120 Participants and procedure

121 Data were obtained from participants in a proof of concept (POC) intervention of 'MyPlan'.

122 'MyPlan' provides personal feedback and helps adults to set and monitor personal and attainable

123 health goals in order to increase either PA level, fruit or vegetable intake. Participants were

124 recruited by distributing flyers to parents of adolescents in secondary schools, by using Facebook

125 and Twitter advertisements and by recruiting university students. Eligible participants were over

12618 years, were able to understand Dutch, and had access to Internet. Potential participants were

127 invited to visit the website. A computer log in system was used to allocate adults to the control or

128 intervention condition. The present study only used data from participants in the intervention

129 group who applied at least one of the self-regulation tools of 'MyPlan'. In the 'MyPlan'

130 intervention programme, adults themselves chose a health behaviour that they wanted to change 
131 (fruit, vegetables or PA), whereafter they filled in online questions about demographic variables

132 (age, gender, socio economic status) and questions about the selected health behaviour. Next, 133 adults had access to the computer-tailored intervention module (T1). After one week (T2) and 134 one month (T3), adults received an email with an invitation for the follow-up modules. These 135 follow-up models evaluated whether they had reached their health goals and whether they 136 attained the recommended health norms. Figure 1 shows the flow of the participants through the 137 intervention modules as a function of the selected health behaviour. The study was approved by 138 the Ghent University Ethics Committee (approval number of the Ghent University Ethics 139 Committee: 670201319313), and an informed consent statement was obtained from each 140 participant.

141 [Insert figure 1 (flow chart) here]

'MyPlan' intervention

143 'MyPlan' is informed by self-regulation and Health Action Process Approach theory. After 144 logging in at the website (www.mijnactieplan.be), participants selected a behaviour of interest 145 (fruit intake, vegetable intake, or PA) and completed the first module for that behaviour, which 146 consisted of several components. Tailored feedback is based upon the answers provided on a questionnaire about the selected behaviour. For PA, the International Physical Activity Questionnaire (IPAQ) was used(Vandelanotte et al. 2005). Tailored feedback consisted of reporting the actual level of PA in different domains (i.e. leisure time PA, active transportation, PA at work, house hold PA), providing feedback about these levels taking into account the health norms, and suggestions to

152 increase PA. For Fruit intake, the average portion of fruit per day was calculated using the 153 Flemish 'Fruit Test'. Participants were asked to indicate how many pieces of each type of fruit 
154 that they ate during the previous week. The average portion of fruit per day was calculated.

155 Participants received a report of this average portion and a comparison of this portion with the

156 health norms. For vegetables, the average grams of vegetables was calculated by means of the

157 'Vegetable Test'(Plaete et al., under review). Participants were asked to indicate the amount of 158 portions of each type of vegetable they ate during the previous week. Average grams of 159 vegetables per day was reported and compared with health norms.

160 Action plans were formulated by answering a series of questions. For example, 161 participants were asked what they wanted to do (e.g. being more physically active by walking), 162 when they wanted to do this (e.g. every Monday evening), where they wanted to do this (e.g. 163 local park), how long they wanted to do this (e.g. 60 minutes) and with whom they wanted to do 164 this (e.g. friends). For PA, adults choose in which domain they wanted to increase their PA level 165 (i.e. leisure time, active life style or both), and defined their goal by selecting activities (e.g. walking, swimming, biking) and by indicating the frequency (days per week) and time (minutes 167 per activity) they wanted to spend on the chosen activity. For fruit and for vegetables, 168 participants indicated the number of days and portions of vegetables they wanted to eat. Next, implementation intentions were stated. Participants were guided to formulate their action plan into an implementation intention plan format (e.g. If it is Monday evening, then I will 171 go to the aerobic lessonsin the local gym). Problem solving was prompted by indicating hindering factors from a predefined list, or -when not listed- by writing down the hindering factors in an open-ended question format.

174 Participants had to reflect upon solutions to overcome these difficulties. This was also done by 175 providing a predefined list of solutions for each hindering factor that could be selected. When not 176 listed, adults could write down their own solutions in an open-ended question format. 

send their action plan to family or friends for social support. physical activity levels, fruit or vegetable intake by using one of the listed possibilities (i.e., personal paper agenda, mobile phone, Excel sheet, online agenda). When module 1 was finished, adults were also invited by email to report their behaviour on the website. Periodic email their goals on the website.

After one week (T2) and one month (T3), participants had access to follow-up modules which assessed whether participants made progress by comparing PA levels/fruit intake/vegetable intake reported at T2/T3 with PA levels/fruit intake/vegetable intake reported at T1. It was also evaluated whether participants reached their goals. Participants could also adapt or maintain their action plan. Action plans could be adapted by stating new goals (easier goals or more difficult goals) and by selecting new difficult situations, hindering factors and solutions. An overview of the intervention programme is given in figure 2 .

192 [Insert figure 2 here]

193

194

195

196

197

\section{Measures}

Demographics

Participants provided information on age, gender and educational level. Participants with a university or college degree were classified as having a 'high educational level' whereas participants with a secondary school degree or lower were classified as having a 'low educational level'. Age was dummy coded into younger adults ( $\leq 40$ years) and older adults ( $>40$ years).

\section{Outcome variables}


200 Goal attainment at T2 and at T3 was operationalised in terms of whether participants attained at

201 least their goal set at T1.

202 Use and feasibility evaluation of behaviour change techniques

203 Participants indicated whether they used particular techniques (selecting hindering factors and 204 solutions, selecting different domains and activities (for PA only), sharing the action plan, 205 monitor behaviour and making a new action plan at T2) (See table 1). These variables were 206 dummy coded into used (1) or not used (0) (See table 1).

207 To evaluate the feasibility of the self-regulation techniques, additional questions were added at

208 the end of the questionnaire in T1. All variables regarding the feasibility of the self-regulation

209 techniques were dummy coded. Table 1 provides an overview of the predictors about the use and

210 feasibility evaluation of the self-regulation techniques.

211 [Insert Table one here.]

\section{Quality of implementation plans}

213 Plan quality of implementation intentions (if-then plans) was evaluated by rating instrumentality

214 and specificity of the plan. We used the rating method of van Osch et al. (2010), rating plans as

215 (1) instrumental or (0) non-instrumental. Plans were rated as instrumental when they were 216 judged to facilitate the chosen behaviour (fruit intake or vegetable intake, PA) and when they 217 were found to be applicable in the situation that was mentioned. The total number of 218 instrumental plans was used for the analysis by dummy coding it into (0) one or two instrumental 219 plans and (1) more than two instrumental plan. Frequent reasons for scoring a plan as not 220 instrumental were nonsense plans, or plans that did not target the chosen behaviour. Non221 instrumental plans were not rated for specificity. Specificity was only scored for plans

222 considered instrumental, and was coded as (0) non-specific, (1) medium specific, and (2) highly 
223 specific. Non-specific plans were vague plans, which were often applicable to various behaviours

224 (e.g. "When it is Friday, I am going to sport"). Plans that were described with a certain amount

225 of detail and direction, but that were still general and applicable to several actions and/or lacked

226 one of the following elements (when, how long and where) were rated as 'medium specific' (e.g.

227 'When I come home after work, I will go playing basket"). Plans were coded as 'highly specific'

228 if a sufficient amount of precision and direction of time (Monday evening 8 am) and place (the

229 local swimming pool) was used and if all elements (when, how long and where) were included

230 (e.g. "When it is Monday evening 8 am, I go swimming for 45 minutes in the local Swimming

231 pool"). Participants had the possibility to make several implementation plans. The mean

232 specificity score of all plans was calculated and used in the analysis by dummy coding it into (0)

233 non-specific plans and (1) medium/highly specific plans. Two researchers independently

234 evaluated all plans on instrumentality and specificity. The interrater reliability was high for

235 instrumentality (Cohen's к 0.89) and substantial for specificity (Cohen’s K=0.76)(Landis \& Koch

236 1977).

\section{Statistical analyses}

238 Baseline characteristics of participants were analysed using descriptive statistics. Logistic

239 regression analyses were performed to predict whether participants reached their goal (= goal

240 attainment) at T2 and T3. Various predictors were taking into account. These included several

241 self-ratings of the feasibility of the self-regulation techniques: the awareness of own behaviour,

242 the motivational value of the personal advice, the instructive value of the personal advice, the

243 motivational value of the action plan, the feasibility of the action plan and the difficulty

244 experienced when making an action plan. Also, selecting hindering factors and solutions,

245 selecting different domains and activities for PA, sharing the action plan, monitor behaviour and 
246 making a new action plan at T2, were added as predictors to take into account the use of these

247 self-regulation techniques. Furthermore, the coded total number of instrumental plans and the

248 mean specificity score of the implementation intention plans were taking into account. All

249 predictors were dummy coded (See Table 1).

250 First we evaluated whether the evaluation of the self-regulation techniques, use of particular self-

251 regulation techniques, and plan quality of implementation intentions predicted whether health

252 goals were attained across the three groups. Next, interaction terms (predictor X behaviour) were

253 included to investigate whether the predictors of goal attainment differed as a function of the

254 chosen behaviour ('PA' or 'fruit and vegetables') of participants. Fruit and vegetables were taken

255 together in one category. In case of a significant interaction effect, the estimated predictive main

256 effect of the predictor only applies to the group that was indicated as the reference category (0).

257 For ease of interpretation, we reported odds ratios and confidence intervals for PA indicated as

258 reference category, and for fruit and vegetables indicated as reference category (See Table 3 and

259 4). Statistical significance was set at a level of 0.05 , p-values between 0.05 and 0.1 were 260 considered borderline significant.

\section{Results}

\section{Baseline characteristics}

263 In the intervention condition, 225 participants started the intervention module for fruit, 84 for 264 vegetables and 267 for PA. Mean age of participants was 30.5 years (SD: 12.5), 39.2\% was male 265 and $72.1 \%$ had a high educational level. Table 2 presents the baseline characteristics for the 266 sample that completed the intervention programme at baseline (T1). Descriptive percentages 267 regarding the use and evaluation of the behaviour change methods are given in Table 1. 
268 In total, 59\% completed module 2 for fruit, 37\% for vegetables and $42 \%$ for PA. Module 3 for

269 was completed by $36 \%$ for fruit, $12 \%$ for vegetables and 17\% for PA. Logistic regression

270 analysis revealed that older participants $(\mathrm{OR}=4.57 ; 95 \% \mathrm{CI}=2.35-8.91 ; \mathrm{p}<0.001)$ and

271 participants with low education $(\mathrm{OR}=1.72 ; 95 \% \mathrm{CI}=1.06-2.78 ; \mathrm{p}=0.028)$ had a significant

272 higher probability for drop-out at follow-up (T3).

273 Goal attainment

274 For all predictors, odds ratios and confidence intervals of the logistic regression analyses are

275 shown in table 3 and 4. In what follows, only significant and borderline significant predictors are 276 reported.

277 Tailored feedback

278 The motivational value of the tailored feedback was a significant predictor of health goal 279 attainment at T2. There was also a borderline significant interaction-effect with behaviour $280(\mathrm{p}=0.090)$, possibly indicating that this only applied for participants in the fruit or vegetable 281 group. Participants in the fruit or vegetable group who perceived the personal advice about fruit 282 or vegetables as motivating were two times more likely to attain their goal at T2 compared to 283 participants in the fruit or vegetable group who did not perceive the personal advice as 284 motivating $(\mathrm{OR}=2.38,95 \% \mathrm{CI}=1.15-4.94 ; \mathrm{p}=0.02)$.

285 Action planning

286 Borderline significance was found for the motivational value of the action plan for health goal 287 attainment at T2. Participants who perceived making their action plan as motivating were more 288 likely to attain their goal at $\mathrm{T} 2$ than participants who did not perceive this as motivating $289(\mathrm{OR}=2.25,95 \% \mathrm{CI}=1.08-4.69 ; \mathrm{p}=0.03)$

290 Problem solving 
291 Selecting hindering factors and solutions was a significant predictor, after including the

292 interaction term with behaviour $(p=0.019)$. Participants in the fruit or vegetable group who

293 selected hindering factors and solutions, were two times more likely to reach their goal at T2

294 compared to participants in the fruit or vegetable group who did not select hindering factors and

295 solutions $(\mathrm{OR}=2.00,95 \% \mathrm{CI}=1.15-3.47 ; \mathrm{p}=0.04)$.

296 Implementation intentions

297 No significant main effects were found for the coded total number of instrumental plans.

298 However, a significant interaction effect was found with behaviour $(\mathrm{p}<0.001)$. Indicating that 299 participants in the fruit or vegetable group who made more than two instrumental 300 implementation plans for fruit or vegetable intake were three times more likely to attain their 301 goals compared to participants in the fruit and vegetable group that made one or two 302 implementation plans $(\mathrm{OR}=1.73,95 \% \mathrm{CI}=1.02-2.96 ; \mathrm{p}=0.09)$. In contrast, participants in the 303 PA group who made one or two instrumental implementation plans for PA were three times 304 more likely to attain their goals compared to participants that made more than two 305 implementation plans for PA $(\mathrm{OR}=0.34,95 \% \mathrm{CI}=0.17-0.64 ; \mathrm{p}==0.006)$. Furthermore, separate 306 analysis solely in the PA group indicated that stating goals in different PA domains (e.g. free 307 time and active living style) was also a significant predictor for PA goal attainment. Participants 308 who stated goals in different PA domains (i.e. goals for their free time and goals for an active 309 living style (e.g. at work) and goals for active transport) were less likely to attain their PA goals 310 compared to participants that stated goals for only one PA domain (i.e. goals for their leisure 311 time only or for active transport only) $(\mathrm{OR}=8.07,95 \% \mathrm{CI}=2.20-29.55 ; \mathrm{p}=0.002)$. The amount of 312 activities selected for goals in the free time could also predict PA goal attainment. Participants 313 who chose two different physical activities (e.g. walking and swimming) were less likely to 
314 attain their PA goals at T2 compares to participants who chose only one activity (OR=0.21, 95\%

$315 \mathrm{CI}=0.08-0.59 ; \mathrm{p}=0.003)$. At $\mathrm{T} 3$, the coded total number of instrumental plans was a significant

316 predictor of health goal attainment, when adjusting for behaviour $(p=0.003)$. Participants in the

317 PA group who made more than two instrumental plans for PA were less likely to succeed in their

318 health goal than participants in the PA group who made one or two instrumental plans $(\mathrm{OR}=0.40$,

$31995 \% \mathrm{CI}=0.16-1.03 ; \mathrm{p}=0.05)$.

320 Borderline significance was found for the mean specificity score of the implementation intention

321 plans for goal attainment at T2. However, the significant interaction-effect with behaviour

$322(\mathrm{p}=0.016)$ indicates that the estimated effect only counts for participants in the fruit or vegetable

323 group. Participants in the fruit or vegetable group who made specific plans were five times more

324 likely to attain their health goal at $\mathrm{T} 2(\mathrm{OR}=4.59,95 \% \mathrm{CI}=1.55-13.63 ; \mathrm{p}<0.021)$.

325 Adapting plans

326 Stating new goals at T2 was found to be a significant predictor of health goal attainment.

327 However, the significant interaction-effect with behaviour indicates that the estimated effect only

328 counts for participants in the fruit or vegetable group. Participants in the fruit and vegetable

329 group who did not state new health goals at T2 were more likely to attain their health goal at T3

330 than participants who stated new goals at $\mathrm{T} 2(\mathrm{OR}=7.54,95 \% \mathrm{CI}=1.96-28.99 ; \mathrm{p}=0.003)$.

\section{Discussion}

332 The results of this study provide further information on how the design, feasibility and 333 applicability of health promotion interventions can be improved to promote optimal behaviour 334 outcomes for different health behaviours. Based on the results, feasible behaviour change 335 techniques can be identified and the content of self-regulation interventions can be improved by 
336 further including and optimizing the different behaviour change techniques that can predict goal

337 attainment.

338 Our study revealed several significant predictors of health goal attainment. After one week, goal

339 attainment was predicted by the motivational value of the personal advice, the motivational value

340 of the action plan, selecting hindering factors and solutions, the specificity score of the

341 implementation intention plans, the coded total number of instrumental plans andselecting

342 different PA activities. After one month, only not stating a new action plan for fruit and

343 vegetables in the follow-up module and making fewer implementation plans for PA could predict

344 health goal attainment. This implicates that perhaps other behaviour change methods or

345 techniques to apply these methods need to be integrated and tested to predict long-term goal

346 attainment.

347 Our results also showed that the efficacy of particular behaviour change techniques varies as a

348 function of type of health behaviour. Some predictors were only significant for fruit and

349 vegetable intake, and other predictors only for PA. The estimated effect of the specificity score

350 of the implementation intention plans, the motivational value of the personal advice, selecting

351 hindering factors and solutions and making a new action plan after one week to attain the health

352 goal was only applicable for participants who chose for fruit or vegetable intake and not for those

353 who chose PA. In line with our results, the meta-analysis of Bélanger-Gravel et al. (2013)

354 revealed 'small-to-medium' effect size of implementation intentions on PA compared to

355 'medium-to-large' effect sizes reported by Gollwitzer and Sheeran (2006) on a variety of health-

356 related behaviours.

357 Moreover, there was one predictor (i.e. the coded total number of instrumental plans) that was

358 positively related to goal attainment for one behaviour, and inversely related for the other health 
359 behaviour. In our study, only a borderline significant effect was found for the coded total number

360 of instrumental plans on fruit and vegetable intake. However, the results for fruit and vegetable

361 intake, are in line with those of Wiedemann, Lippke and Schwarzer (2012), who found that

362 forming a large number of plans may be more effective in changing fruit intake than forming few

363 plans(Wiedemann et al. 2012). Our results imply that 'the more plans, the better' cannot be

364 generalised for all health behaviours. Our study showed opposite results for PA goals. The coded

365 total number of instrumental plans was the only significant predictor for PA goal attainment after

366 one month. Participants in the PA group who made more than two instrumental plans for PA

367 were less likely to succeed in their health goal. Therefore, to increase the chance that adults reach

368 long-term PA goals, our results suggest that PA interventions should be kept simple and focus

369 only on developing a few implementation intentions. The study of Wiedemann et al. (2011) also

370 showed that better intervention effects were associated with two rather than three PA action

371 plans(Wiedemann et al. 2011). Due to our small sample group, we could not investigate the

372 optimal number of plans. Therefore, future research may further focus on the optimal number of

373 plans for different behaviours, especially for PA interventions.

374 In our study, we also conducted separate analyses for the PA group, because participants who

375 choose the PA module had also the opportunity to make plans for the different domains.

376 However, we found that participants who stated goals in different PA domains (i.e. goals for

377 their free time and goals for active transport) were less likely to attain their PA goals compared

378 to participants that stated goals for only one PA domain (i.e. goals for their free time only or 379 goals for active transport only). The amount of activities selected also negatively predicted PA 380 goal attainment. Participants who chose two different physical activities (e.g. walking and 381 swimming) were less likely to attain their PA goals at T2 compared to participants who chose 
382 only one activity. This could perhaps be attributed to the fact that PA is a rather complex

383 behaviour to change(Bélanger-Gravel et al. 2013; De Vet et al. 2009). This also shows that the

384 feasibility of PA goals and plans may be important. However, the feasibility of the action plan

385 was not a significant predictor of PA goal attainment. It may be that adults have difficulties to

386 formulate feasible PA plans, due to the complexity of incorporating PA goals(Bélanger-Gravel et

387 al. 2013; De Vet et al. 2009). Therefore, it would be beneficial to incorporate computerized

388 feedback that gives advice on the feasibility by comparing the current health behaviour with

389 goals (especially for PA). For example, adults who never ran before and who state a plan to run

390 every day for one hour, may better receive feedback about the unfeasibility of their plan. It seems

391 that a small group was already aware that their plans were not feasible, as they indicated this in

392 their evaluation. It may make no sense to pursue such goal, and in such situations adults are

393 probably better prompted to adapt their goals. It may be a good idea to implement an evaluation

394 of the feasibility of plans by participants in eHealth interventions. Another idea is to give

395 participants advice to start with only one or two plans in one PA domain, and to make repeated

396 and/or additional plans after the first goal is achieved.

397 Making implementation intentions of medium to high quality predicted goal attainment. The 398 mean specificity score of the implementation intention plans could only predict goal attainment 399 at short-term (at T2, after one week) in the fruit and vegetable group. In our study, 400 implementation intentions were used to let adults make action plans. Bélanger-Gravel et al. 401 (2013) stated that using implementation intentions for PA only for action planning and not for 402 coping planning (i.e. management of barriers) can decrease the efficacy of implementation 403 intentions. This may explain why the use of implementation intentions could not predict goal 404 achievement for PA goals and after a longer period. We did let participants select 
405

406 in an implementation intention format (i.e. if-then plans) and could also only predict goal

407 attainment in the fruit and vegetable group at short-term. By using the implementation intention

408 format, critical cues in coping plans are linked to the goal-directed behaviour, which creates a

409 strong and automatic cue-response. Previous studies observed the 'if-then' format to yield better

410 behaviour outcomes (Armitage \& Arden 2010; Chapman et al. 2009). Thus, our results

411 strengthen the suggestion of Bélanger-Gravel et al. (2013) to incorporate coping plans into an

412 implementation intention format.

413 Only a small group sent their action plan for social support as part of 'MyPlan', and this could 414

not predict goal attainment. This result shows that further investigation on how to include social support in eHealth interventions is warranted. Morrison et al. (2012) reported in their review study that social context and support mediates eHealth intervention outcomes. To increase intervention effectiveness, they suggest to provide social support by using automated dialogue, peer-to-peer-mediated communication, or information about other real users. Ziegelmann, Lippke, \& Schwarzer (2006) reported more complete action plans and a longer duration of physical activities when participants were assisted by an interviewer trained in motivational interviewing. This suggests that additional personal support by health counsellors trained in motivational interviewing could also lead to additional effects of future planning interventions(De Vet et al. 2009; Ziegelmann et al. 2006).

Making a new action plan in the follow-up module was the only significant predictor for fruit and vegetable attainment after one month. Participants who did not state new health goals at T2 were more likely to attain their health goal at T3. This may indicate that the timing and frequency of follow-up modules might be important to attain health goals at the long-term. 
428 Adults in the fruit and vegetable group who already adapted their plan after one week, had less 429 chance to achieve their goals after one month compared to those who did not adapt their plans 430 yet. In the PA group, adapting plans after one week could not predict goal attainment. This 431 indicates that giving people the possibility to adapt their goals after one week, is maybe too 432 early. To our knowledge, no studies have already investigated the optimal frequency and timing 433 of follow-up modules in self-regulation interventions. Perhaps instructing participants to use 434 follow-up modules at fixed moments is not effective and in contrast to their preference for more 435 flexibility. Therefore, a suggestion for future researchers is to use follow-up modules that are 436 adjustable to the needs of the individual user. Mobile phone apps, for example, make use of real437 time assessment, are constantly accessible and can therefore provide data anywhere and anytime. 438 In this way, tailored feedback and follow-up can be provided at the appropriate time and place, 439 adjusted for individual users(Middelweerd et al. 2014). Using smartphone apps also offers the 440 opportunity for users to monitor their behaviour. Michie et al. (2009) showed that self441 monitoring of behaviour was associated with improved effectiveness of eHealth interventions. 442 However, in our study, prompting monitoring of self-behaviour could not predict health goal 443 attainment. In MyPlan, participants were prompted to monitor their behaviour by asking adults to 444 keep a record of their physical activity levels or fruit and vegetable intake by using a proposed 445 suggestions (e.g. in their personal paper agenda). Furthermore, participants could also 446 monitor/track their behaviour by reporting their behaviour on the website, in the follow-up 447 modules. Perhaps, tools like smart phone apps, in which participants can constantly monitor their 448 behaviour at any place and any time and receive constant feedback on their behaviour change 449 progress, will be perceived as more fun and may be more likely to predict health goal attainment. 
450 After one month, only one significant predictor for fruit and vegetable health goal attainment and

451 one for PA goal attainment could be identified. Our results should be interpreted with caution

452 due to the small sample and high attrition rate, which resulted in a restricted statistical power.

453 This may have influenced the results for the impact on goal attainment at T3. After one month

454 (T3) a notable high attrition rate was observed. Our intervention did contain techniques that have

455 been proposed to enhance sustained use (i.e. goal setting, self-monitoring of behaviour). This is a

456 challenge for many computer-tailored or internet interventions(Schneider et al. 2011), and will

457 need to be addressed in order to use the full potential of eHealth interventions. Perhaps, the time

458 needed (e.g. on average $25 \mathrm{~min}$ ) to complete the first module was too long, or instructions to

459 revisit the website and ways to get access to the follow-up modules were not clear. We only used

460 one email to invite adults to revisit the website. In the future, we may use emails with updated

461 information or an email and SMS reminder system(Schneider et al. 2011). Our drop-out analyses

462 indicated that older participants and participants with lower educational levels had a significant

463 higher probability for drop-out at follow-up (T3). Previous research indicated that participants

464 who complete health interventions tend to be female, middle-aged and high educated(Brouwer et

465 al. 2010; Liang et al. 1999; Schneider 2013). This argues for a further evaluation of strategies to

466 prevent drop-out, especially in low educated and older adults. Also important is that our study

467 was conducted in a rather young (mean age 30,5 years) and low educated population, which may

468 have influenced our results. Therefore, we suggest future research to also try to reach other

469 population groups (e.g. older and low educated adults) when testing eHealth interventions. Due

470 to the low power, we also decided to report borderline significant results. Studies with larger

471 samples are needed to confirm our results. Also, the choice options (e.g. choosing to only form

472 plans for PA in leisure time) have led to some small sample groups, making it not possible to 
473 perform moderator analyses. Next, the short study duration must be taken into account when

474 interpreting the results. As only two significant predictors for health goal attainment at 1 month

475 follow-up could be found, it is important to further identify behaviour change techniques that can

476 predict health goal attainment at 1 month but also at long-term (e.g. after 6 months and 1 year).

477 Therefore, a longer trialwith a larger and robust sample size is needed. Furthermore, it is also

478 important to note that other factors (e.g., quality of theoretical content, combination of behaviour

479 change techniques, participants characteristics) and the combination of factors might also have

480 important effects on intervention outcome and needs further investigation(Michie et al. 2009;

481 Morrison et al. 2012). Finally, future studies should evaluate whether the behaviour change

482 techniques that were theoretically predicted to affect changes in behaviour/health goal attainment

483 can actually influence intervention effectiveness. Therefore, experimental studies with different

484 intervention conditions which do, and do not include sets of behaviour change techniques are

485 needed(Michie et al. 2009).

486 Conclusion

487 To increase the probability that adults attain short-term fruit and vegetable goals, we recommend 488 to integrate (a) personal advice and self-formulated action plans that are evaluated as motivating 489 by participants, (b) a problem solving tool in which adults can select hindering factors and 490 solutions, (c) the recommendation of making multiple implementation plans, (d) 491 instructions/strategies to make specific implementation intentions. To increase the chance that 492 adults reach short-term PA goals, our results suggest that PA interventions should be kept simple 493 and focus only on developing a few implementation intentions. Furthermore, further evaluation 494 of behaviour change techniques (e.g. use of health behaviour apps for self-monitoring of 
495 behaviour and providing real-time follow-up) that can influence long-term goal attainment is 496 necessary. 
497

498

499

500

501

502

503

504

505

506

507

508

509

510

511

512

513

514

515

516

517

518

519

520

521

522

523

524

525

526

527

528

529

530

531

532

533

534

535

536

537

538

539

540

541

542

543

\section{REFERENCES}

Armitage CJ, and Arden MA. 2010. A volitional help sheet to increase physical activity in people with low socioeconomic status: A randomised exploratory trial. Psychol Health 25:1129-1145.

Bélanger-Gravel A, Godin G, and Amireault S. 2013. A meta-analytic review of the effect of implementation intentions on physical activity. Health Psychology Review 7:23-54.

Broekhuizen K, Kroeze W, van Poppel MN, Oenema A, and Brug J. 2012. A systematic review of randomized controlled trials on the effectiveness of computer-tailored physical activity and dietary behavior promotion programs: an update. Ann Behav Med 44:259-286.

Brouwer W, Oenema A, Raat H, Crutzen R, de Nooijer J, de Vries NK, and Brug J. 2010. Characteristics of visitors and revisitors to an Internet-delivered computer-tailored lifestyle intervention implemented for use by the general public. Health Educ Res 25:585-595.

Chapman J, Armitage CJ, and Norman P. 2009. Comparing implementation intention interventions in relation to young adults' intake of fruit and vegetables. Psychol Health 24:317-332.

De Vet E, Oenema A, Sheeran P, and Brug J. 2009. Should implementation intentions interventions be implemented in obesity prevention: the impact of if-then plans on daily physical activity in Dutch adults. Int J Behav Nutr Phys Act 6:11.

De Vries H, and Brug J. 1999. Computer-tailored interventions motivating people to adopt health promoting behaviours: Introduction to a new approach. Patient Education and Counseling 36:99-105.

Gollwitzer P, and Sheeran P. 2006. Implementation Intentions and Goal Achievement: A Meta-analyses of effects and processes. Advances in Experimental Social Psychology 38:69-119.

Hagger MS, Lonsdale A, Koka A, Hein V, Pasi H, Lintunen T, and Chatzisarantis NL. 2012. An intervention to reduce alcohol consumption in undergraduate students using implementation intentions and mental simulations: a cross-national study. Int J Behav Med 19:82-96.

Hallal PC, Andersen LB, Bull FC, Guthold R, Haskell W, and Ekelund U. 2012. Global physical activity levels: surveillance progress, pitfalls, and prospects. The Lancet 380:247-257.

Haskell WL, Lee IM, Pate RR, Powell KE, Blair SN, Franklin BA, Macera CA, Heath GW, Thompson PD, and Bauman A. 2007. Physical activity and public health: updated recommendation for adults from the American College of Sports Medicine and the American Heart Association. Med Sci Sports Exerc 39:1423-1434.

Kroeze W, Werkman A, Brug J. 2006. A Systematic Review of Randomized Trials on the Effectiveness of Computer-Tailored Education on Physical Activity and Dietary Behaviors. Annals of Behavioral Medicine 31(3):205-223.

Landis JR, Koch GG. 1977. The measurement of observer agreement for categorical data. Biometrics 33:159-174.

Lara J, Evans H, O'Brien N, Moynihan P, Meyer D, Adamson A, Errington L, Sniehotta FF, White M, and Mathers J. 2014. Association of behaviour change techniques with effectiveness of dietary interventions among adults of retirement age: a systematic review and meta-analysis of randomised controlled trials. BMC Medicine 12:177.

Liang W, Shediac-Rizkallah M, Celentano D, and Rohde C. 1999. A Population-Based Study of Age and Gender Differences in Patterns of Health-Related Behaviors. Am J Prev Med 17(1).

Lock K, Pomerleau J, Causer L, Altmann D, and McKee M. 2005. The global burden of disease attributable to low consumption of fruit and vegetables: implications for the global strategy on diet. Bulletin of the World Health Organisation 83(2):100-108.

Michie S, Abraham C, Whittington C, and McAteer J. 2009. Effective Techniques in Healthy Eating and Physical Activity Interventions: A Meta-Regression. Health Psychology. 28 (6): 690-701. 
544

545

546

547

548

549

550

551

552

553

554

555

556

557

558

559

560

561

562

563

564

565

566

567

568

569

570

571

572

573

574

575

576

577

578

579

580

581

582

583

584

585

586

587

588

589

590

591
Michie S, Dormandy E, and Marteau TM. 2004. Increasing screening uptake amongst those intending to be screened: the use of action plans. Patient Educ Couns 55:218-222.

Middelweerd A, Mollee JS, van der Wal CN, Brug J, and Te Velde SJ. 2014. Apps to promote physical activity among adults: a review and content analysis. Int J Behav Nutr Phys Act 11:97.

Morrison LG, Yardley L, Powell J, and Michie S. 2012. What design features are used in effective e-health interventions? A review using techniques from Critical Interpretive Synthesis. Telemed J E Health 18:137-144.

Plaete J, De Bourdeaudhuij I, Crombez G, Steenhuyzen S, Dejaegere L, Vanhauwaert E, Verloigne M. Under review. The reliability and validity of short online questionnaires to measure fruit and vegetable intake in adults: the Fruit Test and Vegetable Test. Plos one.

Schneider F. 2013. Reach out and touch? Improving reach and use of an Internet-delivered lifestyle intervention. Maastricht University, School for Public Health and Primary Care: CAPHRI, Department of Health Promotion.

Schneider F, van Osch LA, Kremers SP, Schulz DN, van Adrichem MJ, and de Vries H. 2011. Optimizing diffusion of an online computer tailored lifestyle program: a study protocol. BMC Public Health 11:480.

Schwarzer R. 2008. Modeling Health Behavior Change: How to Predict and Modify the Adoption and Maintenance of Health Behaviors. Applied Psychology 57:1-29.

Sheeran P, Milne S, Webb T, and Gollwitzer P. 2005 Implementation intentions and health behaviour. In: Conner M \& Norman P, editors. Predicting health behaviour. New York: Open University Press 276-323.

Sniehotta FF, Scholz U, and Schwarzer R. 2006. Action plans and coping plans for physical exercise: A longitudinal intervention study in cardiac rehabilitation. Br J Health Psychol 11:23-37.

Spittaels H, De Bourdeaudhuij I, and Vandelanotte C. 2007. Evaluation of a website-delivered computertailored intervention for increasing physical activity in the general population. Prev Med 44:209217.

Springvloet L, Lechner L, and Oenema A. 2014. Planned development and evaluation protocol of two versions of a web-based computer-tailored nutrition education intervention aimed at adults, including cognitive and environmental feedback. BMC Public Health 14:47.

Tones K, and Tilford S. 2001. Effectiveness, Efficiency and Equity, third ed., Nelson Thornes, Cheltenham.

van Genugten L. 2011. Prevention of weight gain among overweight adults: development and evaluation of a computer-tailored self-regulation intervention. PhD thesis. Erasmus University, Department of Public Health.

van Genugten L, van Empelen P, Flink I, and Oenema A. 2010. Systematic development of a selfregulation weight-management intervention for overweight adults. BMC Public Health 10:649.

van Osch L, Lechner L, Reubsaet A, and De Vries H. 2010. From theory to practice: An explorative study into the instrumentality and specificity of implementation intentions. Psychol Health 25:351364.

Vandelanotte C. 2003. Acceptability and feasibility of a computer-tailored physical activity intervention using stages of change: project FAITH. Health Educ Res 18:304-317.

Vandelanotte C, De Bourdeaudhuij I, Philippaerts R, Sjostrom M, and Sallis JF. 2005. Reliability and Validity of a Computerized and Dutch Versionof the International Physical Activity Questionnaire (IPAQ). Journal of Physical Activity and Health 2: 6-75.

WHO. 2003. Diet, Nutrition and the prevention of Chronic diseases, Report of Joint WHO/FAO Expert Consultation.

WHO. 2009. Global Health Risks Mortality: and burden of disease attributable to selected major risks.

WHO. 2010. Global recommendations on physical activity for health. Geneva: World Health Organisation. 
592 Wiedemann AU, Lippke S, Reuter T, Ziegelmann JP, and Schüz B. 2011. The More the Better? The Number of Plans Predicts Health Behaviour Change. Applied Psychology: Health and Well-Being

595 3:87-106.

596

597

Wiedemann AU, Lippke S, and Schwarzer R. 2012. Multiple plans and memory performance: results of a randomized controlled trial targeting fruit and vegetable intake. J Behav Med 35:387-392.

598

Ziegelmann JP, Lippke S, and Schwarzer R. 2006. Adoption and maintenance of physical activity: Planning interventions in young, middle-aged, and older adults. Psychol Health 21:145-163.

599

600

601 


\section{Table 1 (on next page)}

Health behaviour change techniques 


\begin{tabular}{|c|c|c|c|c|}
\hline $\begin{array}{l}\text { Behaviour change } \\
\text { technique }\end{array}$ & Predictor & Question & Values (dummy coded) & n (\%) \\
\hline \multirow[t]{3}{*}{$\begin{array}{l}\text { Tailored feedback } \\
\text { (feasibility evaluation) }\end{array}$} & $\begin{array}{l}\text { The motivational value of the personal } \\
\text { advice }\end{array}$ & $\begin{array}{l}\text { "I Think the personal advice is } \\
\text { motivating" }\end{array}$ & $\begin{array}{l}\text { Personal advice perceived as motivating (1) } \\
\text { Personal advice not perceived as motivating }(0)\end{array}$ & $\begin{array}{l}141(63.2) \\
82(36.8)\end{array}$ \\
\hline & The awareness of own behaviour & $\begin{array}{l}\text { "Did you expect the result of the } \\
\text { personal advice in advance?" }\end{array}$ & $\begin{array}{l}\text { Aware of their behaviour (1) } \\
\text { Not aware of their behaviour (0) }\end{array}$ & $\begin{array}{l}129(57.3) \\
96(42.7)\end{array}$ \\
\hline & The instructive value of the personal advice & $\begin{array}{l}\text { "I Think the personal advice is } \\
\text { instructive" }\end{array}$ & $\begin{array}{l}\text { Personal advice perceived as instructive (1) } \\
\text { Personal advice not perceived as instructive (0) }\end{array}$ & $\begin{array}{l}142(63.7) \\
81(36.3)\end{array}$ \\
\hline $\begin{array}{l}\text { Problem solving } \\
\text { (use) }\end{array}$ & Selected barriers and hindering situations & $\begin{array}{l}\text { "Select those barriers or hindering } \\
\text { situations you want to apply or } \\
\text { formulate it yourself" }\end{array}$ & $\begin{array}{l}\text { No barriers or hindering situations ( } 0 \text { ) } \\
\text { Selected/formulated barriers or hindering situations (1) }\end{array}$ & $\begin{array}{l}126(31.3) \\
277(68.7)\end{array}$ \\
\hline \multirow[t]{3}{*}{$\begin{array}{l}\text { Action planning } \\
\text { (feasibility evaluation) }\end{array}$} & $\begin{array}{l}\text { Perceived difficulty of making an action } \\
\text { plan }\end{array}$ & $\begin{array}{l}\text { "I think it is difficult to make an } \\
\text { action plan" }\end{array}$ & $\begin{array}{l}\text { Perceived making an action plan as difficult (1) } \\
\text { Perceived making an action plan not as difficult (0) }\end{array}$ & $\begin{array}{l}82(37.3) \\
138(62.7)\end{array}$ \\
\hline & The motivational value of the action plan & $\begin{array}{l}\text { "The action plan motivates me to } \\
\text { pursue my goals" }\end{array}$ & $\begin{array}{l}\text { Action plan perceived as motivating (1) } \\
\text { Action plan not perceived as motivating }(0)\end{array}$ & $\begin{array}{l}139(62.9) 82 \\
(37.1)\end{array}$ \\
\hline & The feasibility of the action plan & "My action plan is feasible" & $\begin{array}{l}\text { Action plan perceived as feasible (1) } \\
\text { Action plan not perceived as feasible (0) }\end{array}$ & $\begin{array}{l}217(98.2) \\
4(1.8)\end{array}$ \\
\hline \multirow[t]{2}{*}{ (use) } & Selecting different domains for PA & $\begin{array}{l}\text { "How do you want to improve you } \\
\text { physical activity level?" }\end{array}$ & $\begin{array}{l}\text { By being more active in my free-time }(1) \\
\text { By choosing an active life style }(0)\end{array}$ & $\begin{array}{l}99(54.1) \\
84(45.9)\end{array}$ \\
\hline & Selecting different activities for PA & $\begin{array}{l}\text { "Do you want to select a second } \\
\text { activity for your free time plan?" }\end{array}$ & $\begin{array}{l}\text { Yes, I want to perform a second activity (1) } \\
\text { No, I do not want to perform a second activity (0) }\end{array}$ & $\begin{array}{l}84(54.5) \\
70(45.5)\end{array}$ \\
\hline $\begin{array}{l}\text { Stimulating self- } \\
\text { monitoring } \\
\text { (use) }\end{array}$ & Monitoring behaviour & $\begin{array}{l}\text { "Did you monitor your behaviour the } \\
\text { past week?" }\end{array}$ & $\begin{array}{l}\text { Did monitor behaviour (1) } \\
\text { Did not monitor behaviour }(0)\end{array}$ & $\begin{array}{l}89(39.6) \\
136(60.4)\end{array}$ \\
\hline $\begin{array}{l}\text { Sharing action plan for } \\
\text { social support } \\
\text { (use) }\end{array}$ & Sharing the action plan & $\begin{array}{l}\text { "Select to share your action plan with } \\
\text { friends and family and fill out their } \\
\text { email address" }\end{array}$ & $\begin{array}{l}\text { Sent action plan to family/friends ( } 0) \\
\text { Did not sent action plan to family/friends (1) }\end{array}$ & $\begin{array}{l}57(25.3) \\
168(74.7)\end{array}$ \\
\hline $\begin{array}{l}\text { Goal evaluation and } \\
\text { adjustment } \\
\text { (use) }\end{array}$ & Making a new plan at T2 & Do you want to make a new plan? & $\begin{array}{l}\text { Yes, I want to make a new plan (0) } \\
\text { No, I want to keep the same plan (1) }\end{array}$ & $\begin{array}{l}28(20.4) \\
109(79.6)\end{array}$ \\
\hline
\end{tabular}


Table 2 (on next page)

Baseline characteristics for the total sample and the three conditions separately 


\begin{tabular}{lllll}
\hline & $\begin{array}{l}\text { Total } \\
\text { intervention } \\
\text { group }\end{array}$ & $\begin{array}{l}\text { Intervention } \\
\text { Physical } \\
\text { Activity } \\
(\mathbf{n = 1 5 8})\end{array}$ & $\begin{array}{l}\text { Intervention } \\
\text { Fruit intake }\end{array}$ & $\begin{array}{l}\text { Intervention } \\
\text { Vegetable } \\
\text { intake } \\
(\mathbf{n = 5 0 )}\end{array}$ \\
& (n=166) & \\
\hline Age (years) & $30.5 \pm 12,5$ & $30.5 \pm 12,6$ & $28.1 \pm 10.9$ & $33.8 \pm 13.4$ \\
Gender (\% male) & 39,2 & 44.5 & 47.8 & 33.3 \\
Education level & 72,1 & 73.6 & 75.8 & 66.6 \\
(\% high university or college) & & & & \\
Instrumentality n (\%) & & & & \\
No instrumental plan (N=6) & $6(1.7)$ & $3(1.9)$ & $2(1.4)$ & $1(2.3)$ \\
One instrumental plan (N=159) & $159(45.7)$ & $57(36.3)$ & $60(40.5)$ & $42(97.7)$ \\
Two instrumental plans (N=102) & $102(29.3)$ & $54(34.3)$ & $48(32.4)$ & $0(0)$ \\
Three instrumental plans (N=68) & $68(19.5)$ & $30(19.1)$ & $38(25.7)$ & $0(0)$ \\
Four instrumental plans (N=8) & $8(2.3)$ & $8(5.1)$ & $0(0)$ & $0(0)$ \\
Five instrumental plans (N=3) & $3(0.9)$ & $3(1.9)$ & $0(0)$ & $0(0)$ \\
Six instrumental plans (N=2) & $2(0.6)$ & $2(1.3)$ & $0(0)$ & $0(0)$ \\
Specificity n (\%) & & & & \\
Low specificity (N=28) & $28(8.0)$ & $21(13.0)$ & $3(2.0)$ & $4(9.5)$ \\
Medium specificity (N=219) & $219(62.2)$ & $87(53.7)$ & $98(66.2)$ & $34(81.0)$ \\
High specificity (N=105) & $105(29.8)$ & $54(33.3)$ & $47(31.8)$ & $4(9.5)$ \\
\hline
\end{tabular}




\section{Table 3(on next page)}

Predicting goal attainment at T2

${ }^{\text {aN }}$ o interaction term included for behaviour, with fruit and vegetables as reference category (0); ${ }^{b}$ With included interaction term (predcitorXbehaviour), with fruit and vegetables as reference category (0); ' With included interaction term (predcitorXbehaviour), with physical activity as reference category $(0) * * p<0.05$ : significant predictor; $* p<0.1$ : borderline significant predictor $\mathrm{Cl}$, confidence interval; OR, odds ratio 


\begin{tabular}{|c|c|c|c|c|}
\hline \multirow{3}{*}{ Predictor } & \multicolumn{4}{|c|}{ Goal attainment T2 $(n=274)$} \\
\hline & $\begin{array}{l}\text { Main effect }^{\mathrm{a}} \\
\text { Predictor }\end{array}$ & $\begin{array}{c}\text { Interaction effect } \\
\text { predictor X } \\
\text { behaviour }\end{array}$ & $\begin{array}{l}\text { Main effect } \\
\text { Predictor }\end{array}$ & $\begin{array}{l}\text { Main effect } \\
\text { Predictor }\end{array}$ \\
\hline & $\mathrm{OR}(95 \% \mathrm{CI})$ & (p-value) & $\mathrm{OR}(95 \% \mathrm{CI})$ & $\mathrm{OR}(95 \% \mathrm{CI})$ \\
\hline The motivational value of the personal advice & $1.86(1.06-3.27)^{*}$ & 0.090 & $2.38(1.15,4.94)^{* *}$ & $1,16(0.48,2.78)$ \\
\hline The awareness of own behaviour & $1.22(0.64-2.31)$ & 0.077 & $1.65(0.80-3.40)$ & $0.77(0.33-1.76)$ \\
\hline The instructive value of the personal advice & $0.89(0.47-1.70)$ & 0.045 & $1.20(0.59-2.42)$ & $0.49(0.20-1.19)$ \\
\hline Selecting hindering factors and solutions & $1.45(0.80-2.65)$ & 0.019 & $2.00(1.04-3.85)^{* *}$ & $0.89(0.43-1.86)$ \\
\hline The coded total number of instrumental plans & $0.89(0.52-1.55)$ & $<0.001$ & $1.73(1.02-2.96)^{*}$ & $0.34(0.17-0.64)^{* *}$ \\
\hline The mean specificity score of the implementation intention plans & $3.50(0.97-12.57)^{*}$ & 0.016 & $\begin{array}{c}4.59(1.55- \\
13.63)^{* *}\end{array}$ & $2.20(0.71-6.75)$ \\
\hline The difficulty experienced when making an action plan & $1.22(0.63-2.34)$ & 0.058 & $1.68(0.81-3.49)$ & $0.48(0.15-1.60)$ \\
\hline The motivational value of the action plan & $1.86(1.06-3.27)^{*}$ & 0.210 & $2.25(1.08-4.69)^{* *}$ & $1.34(0.57-3.13)$ \\
\hline The feasibility of the action plan & $1.30(0.62-2.74)$ & 0.516 & $1.06(0.40-2.81)$ & $1.63(0.59-4.51)$ \\
\hline Sharing the action plan & $1.66(0.98-2.79)$ & 0.111 & $1.97(1.06-3.65)^{*}$ & $1.20(0.46-3.16)$ \\
\hline
\end{tabular}


Figure 1 (on next page)

An overview of the intervention programme 


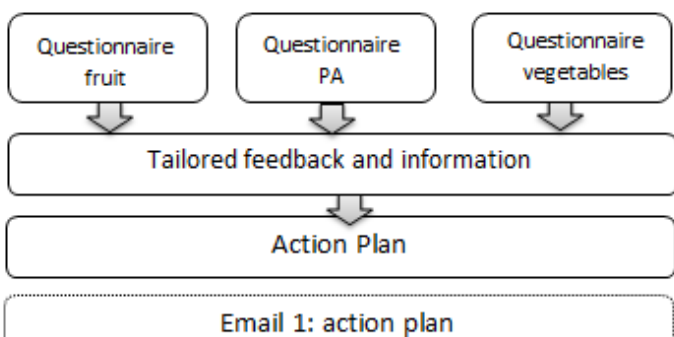

Email 2: invitation for session 2

Iterative feedback and evaluation of action plan

Maintain action plan Adapt action plan

Email 3: action plan

Email 4: invitation for session 3

Iterative feedback and evaluation of action plan 


\section{Table 4 (on next page)}

Predicting goal attainment at T3

${ }^{\text {aN }}$ o interaction term included for behaviour, with fruit and vegetables as reference category (0); ${ }^{b}$ With included interaction term (predcitorXbehaviour), with fruit and vegetables as reference category (0); ' With included interaction term (predcitorXbehaviour), with physical activity as reference category (0)** $p<0.05$ : significant predictor; $* p<0.1$ : borderline significant predictor $\mathrm{Cl}$, confidence interval; OR, odds ratio 


\begin{tabular}{|c|c|c|c|c|}
\hline \multirow{3}{*}{ Predictor } & \multicolumn{4}{|c|}{ Goal attainment $T 3(n=137)$} \\
\hline & $\begin{array}{c}\text { Main effect }^{\mathrm{a}} \\
\text { Predictor } \\
\mathrm{OR}(95 \% \mathrm{CI})\end{array}$ & $\begin{array}{c}\text { Interaction } \\
\text { effect } \\
\text { predictor X } \\
\text { behaviour }\end{array}$ & $\begin{array}{l}\text { Main effect } \\
\text { Predictor } \\
\text { OR }(95 \% \mathrm{CI})\end{array}$ & $\begin{array}{l}\text { Main effect }^{c} \\
\text { Predictor } \\
\text { OR }(95 \% \mathrm{CI})\end{array}$ \\
\hline & & (p-value) & & \\
\hline The motivational value of the personal advice & $1.24(0.55,2.78)$ & 0.230 & $1.52(0.63-3.68)$ & $1.88(0.67,5.30)$ \\
\hline The awareness of own behaviour & $1.09(0.49,2.40)$ & 0.188 & $1.41(0.57,3.45)$ & $0.70(0.26,1.93)$ \\
\hline The instructive value of the personal advice & $0.68(0.29,1.59)$ & 0.101 & $0.38(0.14,1.05)$ & $0.35(0.12,1.04)$ \\
\hline Selecting hindering factors and solutions & $0.97(0.44,2.17)$ & 0.019 & $1.44(0.60,3.47)$ & $0.486(0.18,1.29)$ \\
\hline The coded total number of instrumental plans & $0.99(0.46,2.10)$ & 0.003 & $1.70(0.70,4.11)$ & $0.40(0.16,1.031)^{*}$ \\
\hline The mean specificity score of the implementation intention plans & $1.91(0.41,8.95)$ & 0.035 & $2.57(0.53,12,41)$ & $1.10(0.22,5.57)$ \\
\hline The difficulty experienced when making an action plan & $0.76(0.34,1.69)$ & 0.327 & $0.458(0.096,2.179)$ & $0,41(0.09,1.78)$ \\
\hline The motivational value of the action plan & $1.05(0.46,2.36)$ & 0.228 & $1.29(0.53,3.17)$ & $0.70(0.25,1.96)$ \\
\hline The feasibility of the action plan & $0.66(0.26,1.62)$ & 0.994 & $0.65(0.20,2.18)$ & $0.66(0.20,2.18)$ \\
\hline Sharing the action plan & $1.73(0.74,4.03)$ & 0.243 & $0.40(0.09,1.86)$ & $0.94(0.26,3.36)$ \\
\hline Monitoring between $\mathrm{T} 1$ and $\mathrm{T} 2$ & $1.18(0.57,2.45)$ & 0.618 & $0.74(0.23,2.39)$ & $0.96(0.32,2.84)$ \\
\hline Making a new action plan at $\mathrm{T} 2$ & $4.10(1.33,12.64)^{* *}$ & 0.022 & $7.54(1.96,28.99)^{* *}$ & $1.35(0.34,5.36)$ \\
\hline
\end{tabular}


Figure 2 (on next page)

Flow chart response rate 


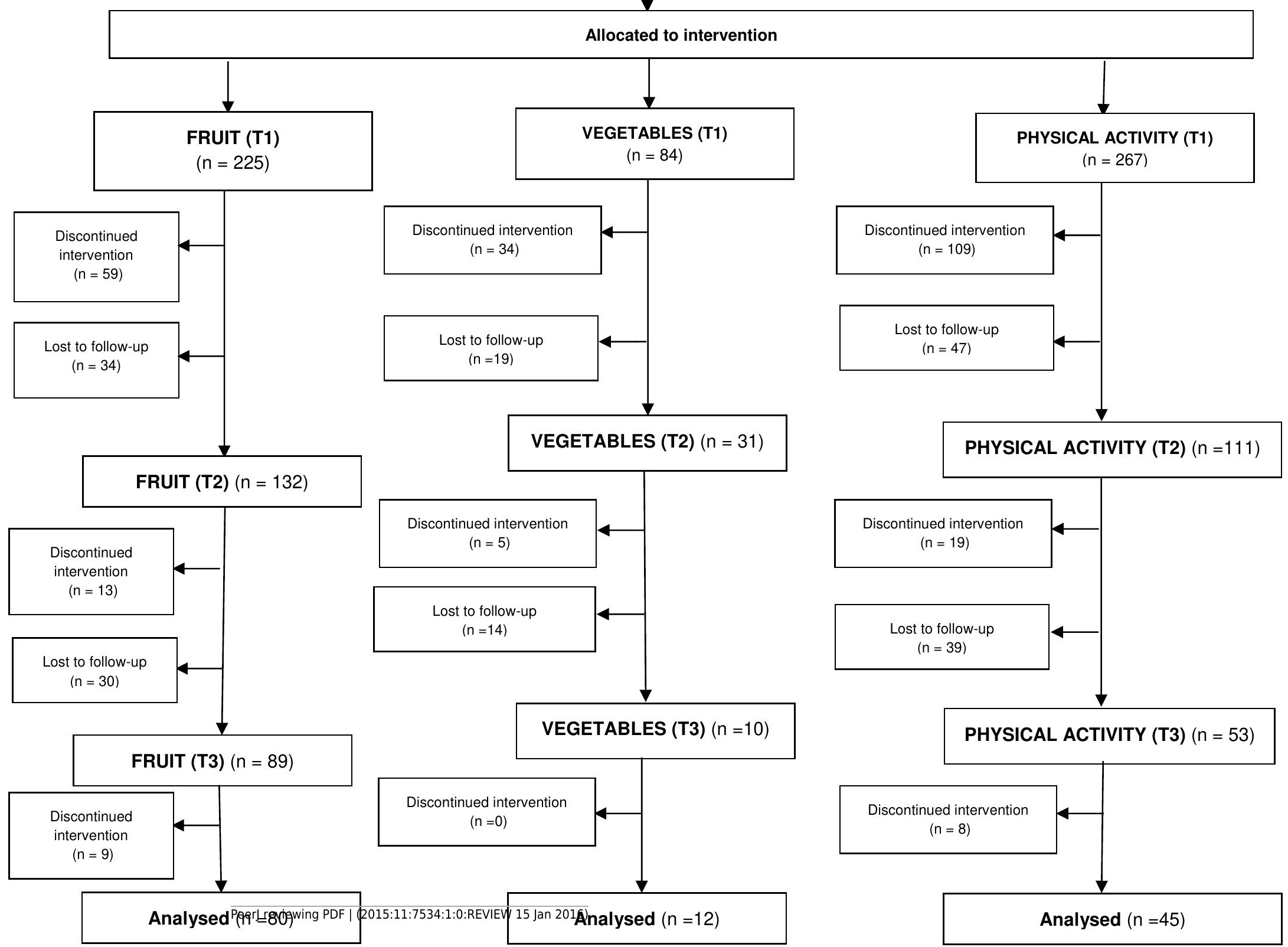

